\title{
Rating players in test match cricket
}

\author{
Sohail Akhtar ${ }^{1 *}$, Philip Scarf ${ }^{2}$ and Zahid Rasool ${ }^{2}$ \\ ${ }^{1}$ University of Malakand, Malakand, Pakistan; and ${ }^{2}$ University of Salford, Salford, UK
}

In general, the evaluation of player performance in test cricket is based on measures such as batting and bowling averages. These measures have a number of limitations, among which is that they fail to take into account the context in which runs are made or conceded and wickets are taken or given away. Furthermore, batting and bowling averages do not allow the comparison of performances in these two disciplines; this is because batting and bowling performances are measured using different metrics. With these issues in mind, we develop a new player rating system for test cricket. We use multinomial logistic regression to model match outcome probabilities session by session. We then use these probabilities to measure the overall contribution of players to the match outcome based on their individual batting, bowling and fielding contributions during each session. Our measure of contribution has the potential for rating players over time and for determining the 'best' player in a match, a series or a calendar year. We use results from 104 matches (2010-2012) to illustrate the method.

Journal of the Operational Research Society advance online publication, 30 April 2014;

doi:10.1057/jors.2014.30

Keywords: ranking; rating; sport

The online version of this article is available Open Access

\section{Introduction}

Rating of teams and players is an important part of most sports. Ratings are used for the selection of teams and players for tournaments, and for seeding players and teams within tournaments (eg, Association of Tennis Professionals (ATP), 2012). They are of interest in their own right (eg, Castrol Football, 2012; FA Premier League (FAPL), 2012; Federation International of Football Association (FIFA), 2012). A rating is a numerical value allocated to each player or team based on their contributions, while a ranking is the ordinal position based on the ratings (Stefani, 2011). Broadly speaking, the rating of teams in team sports and the rating of individual players in individual sports is relatively straightforward and well developed (eg, Stefani, 1977; Elo, 1978; Harville, 1980). Recently, Stefani (2011) reviewed rating systems and discussed the three basic types of sport rating systems, namely, the subjective, objective and accumulative. Typically, objective rating systems use data on results to estimate competitors' strengths. Estimated strengths then directly provide a rating, and rankings can be established from these (eg, Koning, 2009). Many proprietary systems use a combination of the objective and subjective, with results forming the objective component, and the scaling of results according to other factors, such as the importance of a match or tournament, the subjective component (eg, ATP, 2012; FIFA, 2012). The rating of individual players in team sports is more challenging since individual contributions have to be

\footnotetext{
*Correspondence: Sohail Akhtar, Department of Statistics, University of Malakand, Lower Dir 25000, KPK, Pakistan.

E-mail: s.akhtar@uom.edu.pk
}

determined in the presence of a team effect and due to the fact that individuals may make different types of contributions according to their specialisms (McHale et al, 2012). The rating of individual players in test cricket is the focus of this paper.

In test cricket, each team consists of 11 players, a combination of batters and bowlers and a wicket keeper. The standard measures of batting and bowling averages have been used to assess player performance over many years. The Reliance ICC (2011) player rankings are the official ICC rankings, and therein are separate measures for batsmen, bowlers and all-rounders. All-rounders are players who bat and bowl to a frontline standard; all-rounders are rated using a combination of their batting and bowling averages. Interest in estimating measures of player performance in cricket goes back to Johnston et al (1993), who assessed player performance in 1-day cricket using dynamic programming. Beaudoin and Swartz (2003) proposed a new statistic for assessing players' performances in 1-day cricket using the Duckworth/Lewis (D/L) methodology. Lewis (2005) calculates player performance in 1-day cricket. The author also uses the D/L methodology to evaluate players' performances in relation to the runs expected for the stage of the innings and in relation to the resources that are consumed when batting, or contributed when bowling. Lewis (2008) extended this work to evaluate player performance over a longer term. However, test matches are different because in 1-day matches there is no notion of playing out the time remaining for a draw. Recently, Borooah and Mangan (2010) proposed new ways of computing test match batting averages to account for two deficiencies. First, they take account of a player's career consistency. Second, they take account of a player's batting 
Table 1 Batting scorecard for first test, India against Australia in India in October 2010

\begin{tabular}{|c|c|c|c|c|c|}
\hline \multicolumn{3}{|c|}{ Australia batting scorecard } & \multicolumn{3}{|c|}{ India batting scorecard } \\
\hline Players & First innings & Second innings & Players & First innings & Second innings \\
\hline SR Watson & 126 & 56 & G Gambhir & 25 & 0 \\
\hline SM Katich & 6 & 37 & V Sehwag & 59 & 17 \\
\hline RT Ponting & 71 & 4 & R Dravid & 77 & 13 \\
\hline MJ Clarke & 14 & 4 & I Sharma & 18 & 31 \\
\hline MEK Hussey & 17 & 28 & SR Tendulkar & 98 & 38 \\
\hline MJ North & 0 & 10 & SK Raina & 86 & 0 \\
\hline TD Paine $^{\dagger}$ & 92 & 9 & MS Dhoni ${ }^{\ddagger}{ }^{\dagger}$ & 14 & 2 \\
\hline MG Johnson & 47 & 3 & Harbhajan Singh & 0 & 2 \\
\hline NM Hauritz & 9 & 9 & Z Khan & 6 & 10 \\
\hline BW Hilfenhaus & $20 *$ & 6 & VVS Laxman & 2 & $73 *$ \\
\hline DE Bollinger & 0 & $5 *$ & PP Ojha & $0 *$ & $5^{*}$ \\
\hline Extras & 26 & 21 & Extras & 20 & 25 \\
\hline Total & 428 & 192 & & 405 & 216 \\
\hline Wickets & 10 & 10 & & 10 & 9 \\
\hline
\end{tabular}

*denotes a not-out innings.

†wicket keeper.

†team captain.

contribution relative to their team's contribution (total). However, fundamentally these adjustments do not consider the influence of a contribution on match outcome.

We propose a new rating system based on players' performances (batting, bowling and fielding) during each session of a test match. (Note that each day of a 5-day test match is divided into three sessions: morning, afternoon and evening, each nominally $2 \mathrm{~h}$ in length.) There are advantages to looking at players' performances session by session. First, a session-bysession analysis can take account of the state of the batting and bowling conditions; thus, scoring runs on the first day in a match may be quite different from scoring runs on the fourth or fifth day because test pitches deteriorate with the passage of time and batting becomes more difficult in the later stages of the match. Second, a session-by-session analysis can take account of the state of the match (pressure situation); thus, a player who scores 50 runs when his team score is 100 runs for 5 wickets may be making a more valuable contribution and should be rated more highly for this contribution than a player who scores 50 runs when his team score is 100 runs for 1 wicket. A similar argument may be put forward for bowlers and wicket-taking contributions. The system we develop also takes account of the wicket value, that is, whether a wicket of a recognized batsman or 'tail-ender' is taken. Furthermore, we propose a rating system that measures batting and bowling (and fielding) performances on the same scale. Then in principle the rating system can be used to choose the best player in a match or in a series (of matches), or to determine the new emerging player in a particular year, or the best player in a year, whether a batsman, a bowler, an all-rounder (a player who is a frontline batsman and a frontline bowler) or a wicket keeper. Best batting and best bowling performances over a period can also be determined. The system we propose has certain similarities to that developed by Scarf and McHale (2005) and McHale et al (2012) for rating soccer players.

To illustrate the importance of different match contexts, consider the following example: the first test match between India and Australia at Chandigarh, India in 2010. The batting scorecard for this match is shown in Table 1. The match was very closely contested and India won by 1 wicket. Watson scored 126 runs in the first innings and 56 runs in the second innings. Tendulkar scored 98 runs and 38 runs in his two innings. These players scored the maximum number of runs for their respective sides during the match. However, it is difficult to determine how important these batting contributions were in the match from Table 1; this is because the state of the match at the time it was scored is not clear. The session-by-session scorecard (Table 2) shows that Watson and Tendulkar scored runs in relatively easy situations in the match. On the other hand, the most crucial and decisive period appears to be the last four sessions in which Laxman played a very important role, scoring 73 runs and arguably playing the decisive innings for his team in this match. Traditional measures of performance ignore the context of the match, and thus the Reliance ICC player ranking, which is based on traditional measures, does likewise.

In order to take account of the match situation when runs are scored or wickets taken, we analyse the net contribution of players using a model of match outcome probabilities given the position at the end of each session that was proposed by Akhtar and Scarf (2012). This model was developed to predict match outcomes 'in-play'. It is similar in principle to those developed by Brooks et al (2002), Scarf and Shi (2005), Scarf and Akhtar (2011) and Scarf et al (2011a). In particular, Brooks et al (2002) use an ordered probit model to predict match outcome given the batting and bowling strengths at the start of a match, Scarf and Shi (2005) use logistic regression to predict match outcome 
Table 2 Session-by-session scorecard for match in Table 1

\begin{tabular}{|c|c|c|c|c|c|c|c|c|c|c|c|c|c|c|}
\hline \multirow[b]{2}{*}{ Session } & \multicolumn{3}{|c|}{ Day 1} & \multicolumn{3}{|c|}{ Day 2} & \multicolumn{3}{|c|}{ Day 3} & \multicolumn{3}{|c|}{ Day 4} & \multicolumn{2}{|c|}{ Day 5} \\
\hline & At lunch & At tea & End & At lunch & At tea & End & At lunch & At tea & End & At lunch & At tea & End & At lunch & At tea \\
\hline Lead & 101 & 179 & 224 & 295 & 390 & 318 & 237 & 148 & 23 & 123 & 188 & 160 & 53 & -1 \\
\hline$W_{1}$ & 1 & 3 & 5 & 6 & 8 & 10 & 10 & 10 & 10 & 13 & 16 & 20 & 20 & 20 \\
\hline$W_{2}$ & 0 & 0 & 0 & 0 & 0 & 2 & 3 & 4 & 10 & 10 & 10 & 14 & 18 & 19 \\
\hline
\end{tabular}

$W_{1}$ is Australia total wickets down at end of session and $W_{2}$ is India total wickets down at end of session.

given the end of third innings position, Scarf and Akhtar (2011) extend this latter work to the end of first and second innings positions, and finally Scarf et al (2011a) consider declaration strategy using the third innings position model.

Using the session-by-session match outcome probabilities, we measure the effect of a contribution (runs scored or wickets taken during a session) on the match outcome probabilities; the greater the effect, the greater the value of the contribution and the more highly the contribution should be rated in the rating system. This principle of rewarding contributions by players in a team according to the effect of the contribution on the match outcome was essentially put forward in the development of the EA Sports Player Performance Index for soccer (McHale et al, 2012). The novelty of our paper lies in implementing this principle in the context of a sport in which players make fundamentally different kinds of contributions. Early development of the work is described in Scarf et al (2011b) and Akhtar (2011).

The structure of the paper is as follows. The session-bysession prediction model is briefly described. We then describe how the prediction model is used to determine player contributions. We then compare different potential method measures of player contribution. Furthermore, we illustrate player rating with examples. Lastly, we introduce a weighted average rating scheme.

\section{Measuring batting, bowling and fielding contributions}

We use match outcome probabilities to measure the contributions of players. The probabilities are predicted using a nominal multinomial logistic regression fitted session by session to data on 146 test matches. Data were obtained from the ESPNcricinfo website (ESPN Cricinfo, 2010), and the complete data set of 146 matches relates to all the test matches in the period between November 2005 and March 2010, excluding those matches for which the session-by-session data were not available and in which more than 90 overs were lost to poor weather. Sessionby-session information is not generally available before November 2005. The data were processed in order to obtain session-by-session information (eg, Table 2) for each match.

The nominal multinomial logistic regression model is as follows. With match outcome $Y$ taking values $(1,0,-1)$ to denote a win, draw and loss, respectively, covariates denoted by $X$ and taking a draw (0) as a reference category, the model
Table 3 Best fitting multinomial logistic regression model at each stage, and showing Nagelkerke $R^{2}$ and number of matches used to estimate the model

\begin{tabular}{lllcc}
\hline Day & Session & Model & $\begin{array}{c}\text { Nagelkerke } \\
R^{2} \text { (in } \\
\text { percentage) }\end{array}$ & $\begin{array}{c}\text { Number } \\
\text { of matches }\end{array}$ \\
\hline Day 1 & At lunch & $G+L+W_{1}$ & 24.4 & 146 \\
& At tea & $G+L+W_{1}$ & 32.3 & 146 \\
& End of day & $G+L+W_{1}$ & 39.6 & 146 \\
Day 2 & At lunch & $G+L+W_{1}+W_{2}$ & 44.0 & 146 \\
& At tea & $G+L+W_{1}+W_{2}$ & 53.4 & 146 \\
& End of day & $G+L+W_{1}+W_{2}$ & 56.7 & 146 \\
Day 3 & At lunch & $G+L+W_{1}+W_{2}$ & 60.7 & 146 \\
& At tea & $G+L+W_{1}+W_{2}$ & 66.5 & 146 \\
& End of day & $G+L+W_{1}+W_{2}$ & 72.7 & 143 \\
Day 4 & At lunch & $G+L+W_{1}+W_{2}$ & 74.8 & 131 \\
& At tea & $L+W_{1}+W_{2}$ & 74.2 & 122 \\
& End of day & $L+W_{1}+W_{2}$ & 80.5 & 111 \\
Day 5 & At lunch & $L+W_{1}+W_{2}$ & 84.0 & 91 \\
& At tea & $L+W_{1}+W_{2}$ & 94.9 & 76 \\
\hline
\end{tabular}

Covariates here are $L$, lead of reference team; $G$, ground effect; $W_{1}$, total wickets down by reference team; $W_{2}$, total wickets down by opponents.

assumes $Y$ has a multinomial distribution, that is, $Y \sim M N$ $\left(p_{1}, p_{0}, p_{-1} ; \sum p_{i}=1\right)$, where $p_{1}, p_{0}$ and $p_{-1}$ represent the probability of a win, a draw and a loss, with

$$
\begin{aligned}
p_{1} & =P(Y=1 \mid X) \\
& =\frac{\exp \left(\alpha_{1}+\beta_{1}^{T} X\right)}{\left\{1+\exp \left(\alpha_{1}+\beta_{1}^{T} X\right)+\exp \left(\alpha_{-1}+\beta_{-1}^{T} X\right)\right\},} \\
p_{0} & =P(Y=0 \mid X) \\
& =\frac{1}{\left\{1+\exp \left(\alpha_{1}+\beta_{1}^{T} X\right)+\exp \left(\alpha_{-1}+\beta_{-1}^{T} X\right)\right\},} \\
p_{-1} & =P(Y=-1 \mid X) \\
& =\frac{\exp \left(\alpha_{-1}+\beta_{-1}^{T} X\right)}{\left\{1+\exp \left(\alpha_{1}+\beta_{1}^{T} X\right)+\exp \left(\alpha_{-1}+\beta_{-1}^{T} X\right)\right\} .}
\end{aligned}
$$

A sequence of models with covariates lead, ground effect and total wickets lost for each team is obtained. These models are given in Table 3 . The coefficients are shown in 
Table 4 The fitted parameter estimates for each session of the match with covariates lead $(L)$, total wickets down by reference team $\left(W_{1}\right)$, total wickets down opposing team $\left(W_{2}\right)$ and ground effect $(G)$

\begin{tabular}{|c|c|c|c|c|c|c|c|c|c|c|c|}
\hline & & \multicolumn{5}{|c|}{ Win/draw } & \multicolumn{5}{|c|}{ Loss/draw } \\
\hline & & Constant & $G$ & $L$ & $W_{1}$ & $W_{2}$ & Constant & $G$ & $L$ & $W_{1}$ & $W_{2}$ \\
\hline \multirow[t]{3}{*}{ Day 1} & Lunch & 2.719 & -3.485 & -0.009 & -0.062 & - & 1.839 & -4.977 & -0.012 & 0.504 & - \\
\hline & Tea & 1.977 & -3.830 & -0.004 & 0.196 & - & 1.042 & -5.345 & -0.007 & 0.578 & - \\
\hline & End & 2.566 & -3.815 & -0.005 & 0.139 & - & 2.551 & -4.952 & -0.012 & 0.394 & - \\
\hline \multirow[t]{3}{*}{ Day 2} & Lunch & 2.562 & -3.712 & -0.003 & 0.027 & 0.161 & 3.454 & -5.648 & -0.012 & 0.263 & -0.119 \\
\hline & Tea & -0.262 & -3.766 & 0.001 & 0.183 & 0.236 & 0.447 & -5.585 & -0.011 & 0.496 & -0.146 \\
\hline & End & 0.126 & -4.238 & 0.001 & 0.050 & 0.310 & 0.656 & -5.414 & -0.010 & 0.300 & 0.037 \\
\hline \multirow[t]{3}{*}{ Day 3} & Lunch & -1.461 & -4.467 & 0.002 & 0.129 & 0.298 & -0.932 & -5.957 & -0.010 & 0.393 & 0.052 \\
\hline & Tea & -2.990 & -4.661 & 0.004 & 0.067 & 0.485 & -3.334 & -4.620 & -0.009 & 0.421 & 0.179 \\
\hline & End & -6.920 & -4.288 & 0.002 & 0.260 & 0.583 & -6.100 & -4.947 & -0.010 & 0.669 & 0.088 \\
\hline \multirow[t]{3}{*}{ Day 4} & Lunch & -8.926 & -3.404 & 0.003 & 0.168 & 0.759 & -7.823 & -4.690 & -0.012 & 0.695 & 0.124 \\
\hline & Tea & -9.260 & - & 0.003 & 0.176 & 0.569 & -8.690 & - & -0.015 & 0.899 & -0.341 \\
\hline & End & -11.614 & - & -0.001 & 0.323 & 0.554 & -16.800 & - & -0.025 & 1.370 & -0.330 \\
\hline \multirow[t]{3}{*}{ Day 5} & Lunch & -20.321 & - & -0.007 & 0.617 & 0.759 & -23.964 & - & -0.031 & 1.653 & -0.223 \\
\hline & Tea & -130.711 & - & -0.025 & 5.896 & 1.154 & -404.939 & - & -0.302 & 24.858 & -4.288 \\
\hline & End & -130.711 & - & -0.025 & 5.896 & 1.154 & -404.939 & - & -0.302 & 24.858 & -4.288 \\
\hline
\end{tabular}

Table 4. There are two points to note here. First, the win-draw comparison and the loss-draw comparison depend on the covariates in different ways-this is a property of nominal as opposed to ordinal multinomial logistic regression. Second, the wickets covariate is transformed to wicket resources before fitting-this allows the value of the tenth wicket, for example, to be less than that of the first wicket in the wickets-lost effect. The models of Akhtar and Scarf (2012) included further covariates: pre-match team strengths and home advantage. We do not use these here because our view is that the worth of the player should be based on the current performance. Finally, note that the number of matches available for model fitting for later sessions decreases because not all matches last the full 5 days.

Using these models, we can predict the match outcome probabilities (win, draw and loss) given the match position at the end of each session $t(t=1,2,3 \ldots 15)$. These are given by

$$
P_{t}^{A}(y)=P_{t}\left(Y=y \mid L=l_{t}, W_{1}=w_{1, t}, W_{2}=w_{2, t}\right)
$$

where $P_{t}^{A}$ denotes the probabilities at the end of session $t$, and $W_{1}, W_{2}$ and $L$ are, respectively, the total wickets down of the reference team, the total wickets down of the opponent team and the lead of the reference team. The reference team bat first in the match. The actual match position at the end of session $t$ is $\left(L=l_{t}, W_{1}=w_{1, t}, W_{2}=w_{2, t}\right)$.

Next, to measure performance session by session, we define hypothetical positions for the batting team and for the bowling team. For the batting team, we define a hypothetical position at (the end of) session $t$ in which batsmen have not scored any runs in session $t$, and obtain match outcome probabilities given the hypothetical position:

$$
P_{t, b a t}^{H}(y)=P_{t}\left(Y=y \mid L=l_{t-1}, W_{1}=w_{1, t}, W_{2}=w_{2, t}\right) .
$$

Thus, the hypothetical position for the batting side at the end of session $t$ is $\left(L=l_{t-1}, W_{1}=w_{1, t}, W_{2}=w_{2, t}\right)$; if the batters score nothing in the session then their lead does not increase and remains at $l_{t-1}$, which is the lead at the end of the previous session $t-1$.

Similarly for the bowling side, we define a hypothetical position at the end of session $t$ in which bowlers have not taken any wickets in session $t$ and obtain match outcome probabilities given the hypothetical position

$$
P_{t, b o w l}^{H}(y)=P_{t}\left(Y=y \mid L=l_{t}, W_{1}=w_{1, t-1}, W_{2}=w_{2, t-1}\right) .
$$

Thus, the hypothetical position for the bowling team in session $t$ is $\left(L=l_{t}, W_{1}=w_{1, t-1}, W_{2}=w_{2, t-1}\right)$. In this way, if the bowling team take no wickets in the session then the wickets down for the other team remains at $w_{t-1}$. Of course, the wickets down for the bowling team remains unchanged during the session.

At the end of each session, to assess the worth of players' contributions, we use the difference between the hypothetical match outcome probabilities and the actual match outcome probabilities. These differences, for batters and for bowlers, are our measures of total player contribution during the session. The probability difference (total contribution) is then awarded to a batter according to his share of the runs scored in the session, and to a bowler according to his share of wickets taken in the session. For bowling contributions, however, a share is also given to fielders. The details of these procedures are given below.

However, matters are not straightforward. This is because we can look at probabilities in relation to alternative outcomes: winning, drawing or at least one of these, or equivalently, not losing. Hence, we can calculate in the manner described above the value of a contribution to winning or the value of a contribution to not losing. Different situations have been 
a

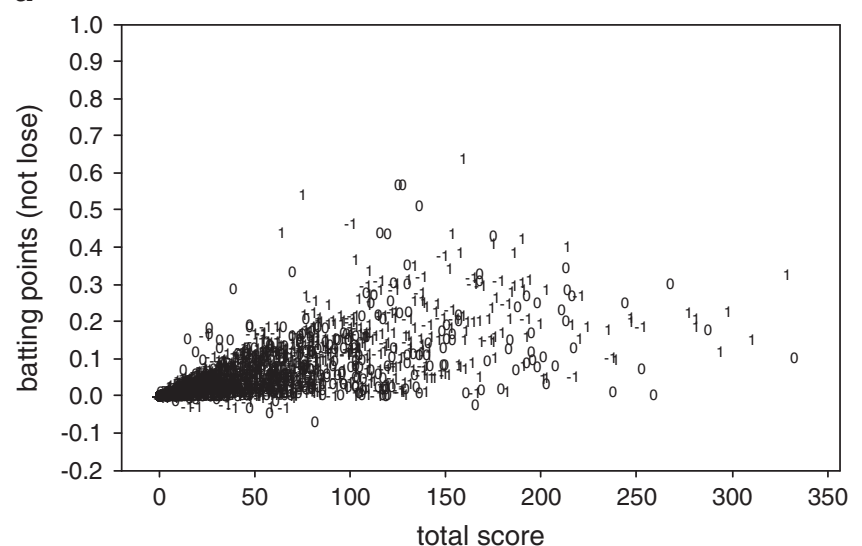

C

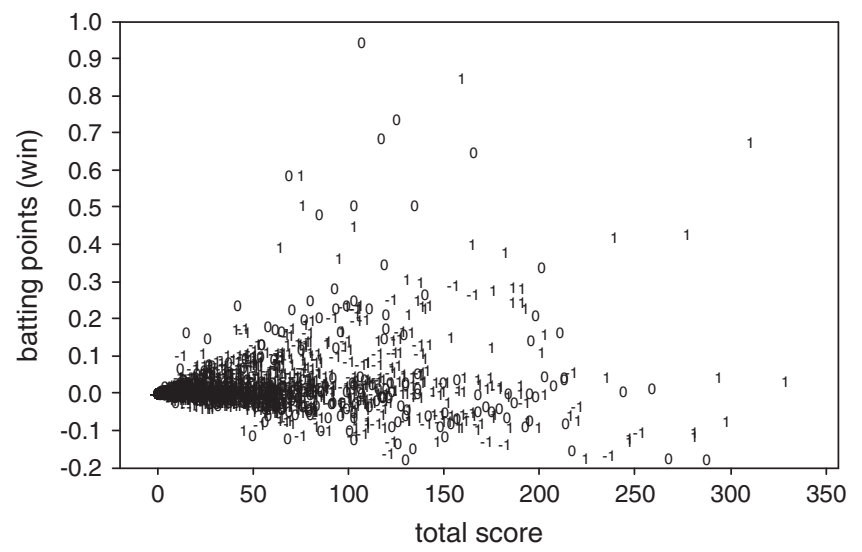

b

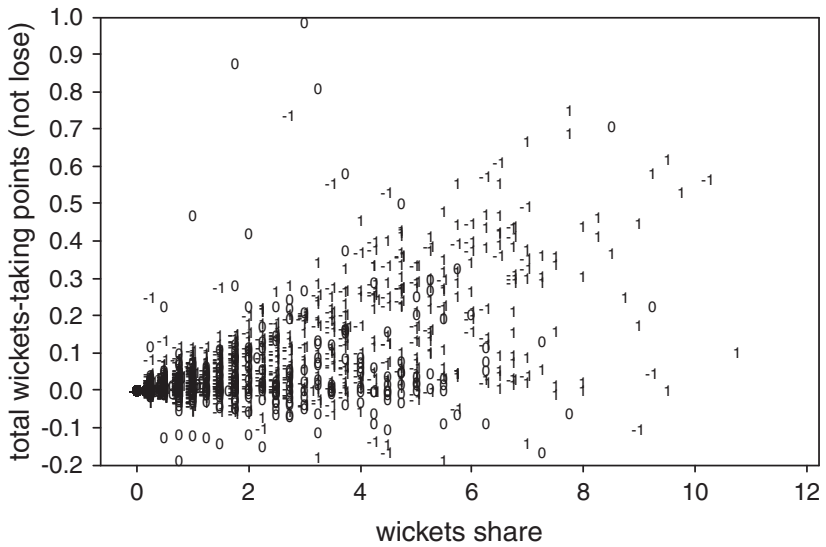

d

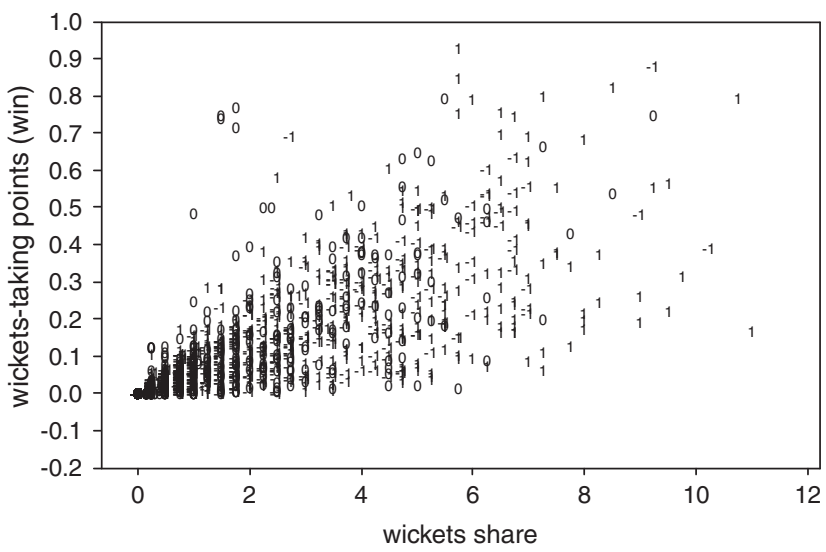

Figure 1 For individual players: (a) total batting points (not lose criterion) versus runs in the match; (b) total wicket-taking points (not lose criterion) versus wickets share; (c) total batting points (win criterion) versus runs in the match; (d) total wicket-taking points (win criterion) versus wickets share; 1 (win), 0 (draw), -1 (loss) from point of view batting and bowling team; respectively (104 matches).

investigated, and we propose one criterion for batters and another for bowlers. For batters, we look at probability differences with respect to 'not losing' and at bowlers with respect to winning. We believe that looking at not losing is a reasonable approach to rate the batters in test cricket. This is because batting teams act conservatively and early in a game will aim to bat towards a position from which they cannot lose. Even later in a game, a team will bat for a win only if a target or a lead is moderate; otherwise they play for a draw. On the other hand, bowlers and fielders predominantly play for a win - the notion of bowlers and fielders playing for a draw does not really exist, despite the fact that fields might be set to be defensive. The numerical results considered later in Section 4 (Figure 1) provide a further justification of these choices of criteria. Alternatively, one might use an expected 'points' gained criterion for both batters and wicket takers that assumes 1 point for a win and 2 points for a draw.

The value of a player's contribution is computed at the end of each session in the following way. Defining the event $\{w i n\}$ that the match outcome is a win for the reference side, and likewise for $\{$ lose $\}$, and $\{$ not lose $\}=\{$ win $\cup$ draw $\}$, the batting and bowling contributions of all players in session $t$ are

$$
\begin{aligned}
C_{t, b a t} & =P_{t}^{A}(\{\text { not lose }\})-P_{t, \text { bat }}^{H}(\{\text { not lose }\}) \\
C_{t, \text { bowl }} & =P_{t}^{A}(\{\text { win }\})-P_{t, \text { bowl }}^{H}(\{\text { win }\}) .
\end{aligned}
$$

The value of an individual player's batting contribution is

$$
C_{i, t, b a t}=C_{t, b a t} \times\left(\frac{r_{i, t}}{r_{t}}\right) .
$$

where $r_{i, t}$ is the runs scored by player $i$ in session $t$ and $r_{t}$ is the total runs scored by his team in session $t$.

For wickets taken, we proceed in the same way in principle to runs scored, sharing the total points among those who take wickets. However, we make allowances for fielding contributions (catches, run-outs and stumpings), and award a share of the points for a wicket to the fielder if indeed a fielder contributed. The choice of the number to express such a share for catches and stumpings is arbitrary. We suppose there are $j=1, \ldots, n$ types of wicket-taking contribution, each of which has share $\alpha_{j}$ of the points. Thus, $j=1$ corresponds to a wicket 
taken by the bowler (bowled, lbw, $\alpha_{1}=1$ ), $j=2$ corresponds to a catch taken by a fielder $\left(\alpha_{2}=0.25\right)$ and $j=3$ corresponds to the bowler contribution in this case $\left(\alpha_{3}=0.75\right)$. Run-out contributions are shared between the fielders involved. Stumpings are treated similarly to catches. For player $i$ in session $t$, the number of contributions of type $j, z_{i t j}$, can be obtained from the scorecard. If the total number of wickets taken in session $t$ is $z_{t}$ then the wicket-taking points awarded to player $i$ in session $t$ is

$$
C_{i, t, b o w l}=C_{t, b o w l} \times \frac{\sum_{j=1}^{n} z_{i t j} \alpha_{j}}{z_{t}} .
$$

The net contribution of player $i$ in the match is then the sum of contributions from all sessions:

$$
\sum_{t=1}^{15}\left(C_{i, t, b o w l}+C_{i, t, b a t}\right) .
$$

We assume in these expressions that there is no change of innings during a session. When there is, we calculate the actual and hypothetical probabilities in a slightly different way. We suppose in effect that the within-session change of innings occurs at a virtual session-end. The outcome probabilities for the virtual session-end are calculated by interpolating between the probabilities that can be calculated for the actual sessionends immediately before and after the virtual session-end. We carry out the interpolation linearly with weights determined by the number of overs into the session at which the change of innings occurs. We omit the details of the calculation here. A player will only score batting and wicket-taking points in the same session if there is a change of innings in the session. The following should also be noted. First, in modelling match outcome, lead is calculated session by session throughout the match with respect to the reference team, the team batting first in the match. Therefore, all the above formulae are strictly applicable to the reference team. For the opponents, the team bowling first, the bowling criterion $\{$ win $\}$ of the reference team becomes $\{$ lose $\}$ of the reference team and vice versa. Second, the change in the probabilities at the end of a match (last session) is calculated by comparison of the hypothetical position with the actual match result. Third, we could use different criteria, for example, $\{$ win $\}$ for batters and $\{$ not lose $\}$ for bowlers. This is explored in the example below. Finally, our intention is to produce a rating system where total batting and wicket-taking points over all time should be the same. However, we are not yet in a position to do this as we have not collected data on sufficient matches.

\section{Comparison of potential measures of contribution}

We now compare the approaches described above. Batting and wicket-taking points for individual players are calculated by the above stated approaches, and plotted in Figure 1, for 104 test matches played in 2010, 2011 and 2012. To calculate the ratings of not too large a number of players for a reasonably large number of matches, we only consider the top eight teams
(Australia, New Zealand, Pakistan, India, South Africa, West Indies, Sri Lanka and England). Figure 1(a) clearly shows a positive relationship between batting points under the not lose criterion and total score. On the other hand, using the $\{w i n\}$ criterion for batters, some of the players earn negative points (Figure 1(c)). This is because batters do not always play for a win; sometimes batters bat slowly for a draw when the opponent's lead is large. When a batter bats slowly the win and loss probabilities decrease and the draw probability increases. Therefore, those batters who play for a draw can receive negative points under the $\{$ win $\}$ criterion. By using the $\{$ not lose $\}$ criterion for wicket taking (Figure 1(b)), some players received negative points. On the other hand, Figure $1(\mathrm{~d})(\{$ win $\}$ criterion for wicket-taking points) gives a stronger positive relationship. Thus, these plots would appear to support the idea of using the $\{$ win $\}$ criterion for wicket-takers and the $\{$ not lose $\}$ criterion for batting points.

\section{Examples}

\subsection{Example 1}

Consider now the third test between Australia and England, Perth, 2010. Table 5 provides the summary of players' batting, bowling and fielding contributions in the match. Batting contributions use $\{$ not lose $\}$ and bowling and fielding contributions use $\{$ win $\}$. Australia batted first, scoring 268 runs in their first innings and 309 runs in their second innings. In reply, England scored 187 runs in their first and 123 runs in the second innings. Australia won this match by 267 runs. Hussey scored maximum runs in the match and received maximum batting points (0.53). On the other hand, Johnson scored 63 runs and took nine wickets in the match. He received the maximum points (0.66) with this all-round performance and was declared man of the match. Note that wicket-taking points are adjusted (rescaled) so that total batting points and total wicket-taking points are equal; we do this to standardize the contributions. Such standardization might be carried out over a series of matches, or even over all matches played in a specified period. The rationale for this is that ultimately, if enough matches are considered, run-scoring and wicket-taking contributions ought to be balanced.

\subsection{Example 2}

Consider now the 2010/2011 Ashes, a five-match test series between England and Australia played in Australia. The results of adopting the contribution system are shown in Table 6. This series was an interesting one for all stakeholders: fans, teams, bookmakers and the communication-media organizations. Squads for each team contained up to 17 players so that more than 22 players took part in at least one match. For brevity, only the top 20 players are presented. Note also that wicket-taking points are rescaled so that total batting points and total wickettaking points over the series are equal in order to standardize the 
Table 5 Player contributions in the third Ashes test, Australia versus England, Perth, 2010

\begin{tabular}{|c|c|c|c|c|c|c|c|c|c|c|}
\hline & Runs & $\begin{array}{l}\text { Wickets } \\
\text { share }\end{array}$ & $\begin{array}{l}\text { Run outs } \\
\text { (fielding) }\end{array}$ & $\begin{array}{l}\text { Catches } \\
\text { (fielding) }\end{array}$ & $\begin{array}{l}\text { Fielding } \\
\text { share }\end{array}$ & $\begin{array}{c}\text { Batting } \\
\text { points }\end{array}$ & $\begin{array}{c}\text { Wicket-taking } \\
\text { points }\end{array}$ & $\begin{array}{l}\text { Fielding } \\
\text { points }\end{array}$ & $\begin{array}{c}\text { Total } \\
\text { points }\end{array}$ & Rank \\
\hline \multicolumn{11}{|l|}{ Australia } \\
\hline Watson & 108 & 0 & 0 & 2 & 0.5 & 0.42 & 0.00 & 0.04 & 0.46 & 3 \\
\hline Hughes & 14 & 0 & 0 & 0 & 0 & 0.06 & 0.00 & 0.00 & 0.06 & 20 \\
\hline Ponting(*) & 13 & 0 & 0 & 2 & 0.5 & 0.01 & 0.00 & 0.04 & 0.05 & 21 \\
\hline Hussey & 177 & 0 & 0 & 2 & 0.5 & 0.53 & 0.00 & 0.02 & 0.55 & 2 \\
\hline Clarke & 24 & 0 & 0 & 0 & 0 & 0.10 & 0.00 & 0.00 & 0.10 & 18 \\
\hline Smith & 43 & 0 & 0 & 2 & 0.5 & 0.15 & 0.00 & 0.03 & 0.18 & 12 \\
\hline Haddin(+) & 60 & 0 & 0 & 3 & 0.75 & 0.11 & 0.00 & 0.06 & 0.16 & 14 \\
\hline Johnson & 63 & 8 & 0 & 0 & 0 & 0.15 & 0.51 & 0.00 & 0.66 & 1 \\
\hline Harris & 4 & 7.5 & 0 & 0 & 0 & 0.01 & 0.40 & 0.00 & 0.41 & 4 \\
\hline Siddle & 43 & 1 & 0 & 0 & 0 & 0.13 & 0.09 & 0.00 & 0.21 & 9 \\
\hline Hilfenhaus & 13 & 0.75 & 0 & 0 & 0 & 0.04 & 0.07 & 0.00 & 0.10 & 16 \\
\hline \multicolumn{11}{|l|}{ England } \\
\hline Strauss(*) & 67 & 0 & 0 & 1 & 0.25 & 0.26 & 0.00 & 0.03 & 0.28 & 6 \\
\hline Cook & 45 & 0 & 0 & 1 & 0.25 & 0.15 & 0.00 & 0.02 & 0.17 & 13 \\
\hline Trott & 35 & 0 & 0 & 0 & 0 & 0.06 & 0.00 & 0.00 & 0.06 & 19 \\
\hline Pietersen & 3 & 0 & 0 & 0 & 0 & 0.00 & 0.00 & 0.00 & 0.00 & 22 \\
\hline Collingwood & 16 & 0.75 & 0 & 3 & 0.75 & 0.04 & 0.02 & 0.04 & 0.10 & 17 \\
\hline Bell & 69 & 0 & 0 & 2 & 0.5 & 0.19 & 0.00 & 0.01 & 0.20 & 10 \\
\hline Prior(+) & 22 & 0 & 0 & 1 & 1 & 0.04 & 0.00 & 0.07 & 0.11 & 15 \\
\hline Swann & 20 & 1.5 & 0 & 2 & 0.5 & 0.03 & 0.13 & 0.02 & 0.18 & 11 \\
\hline Tremlett & 3 & 7 & 0 & 0 & 0 & 0.01 & 0.40 & 0.00 & 0.41 & 5 \\
\hline Anderson & 3 & 3.25 & 0 & 1 & 0.25 & 0.00 & 0.23 & 0.02 & 0.24 & 7 \\
\hline Finn & 3 & 4 & 0 & 0 & 0 & 0.00 & 0.24 & 0.00 & 0.24 & 8 \\
\hline substitute & 0 & 0 & 0 & 0 & 0 & 0.00 & & & 0.00 & \\
\hline
\end{tabular}

contributions. Using the combined contribution measure to evaluate the player of the series or tournament award, we see that Alastair Cook, the England opening batsman, is placed in top position. Behind him in second is his countryman Anderson, a bowler whose contribution with the ball was also outstanding. It is difficult to argue against these players being the best batsman and bowler, respectively. Cook in fact was awarded the man of the series accolade. England players occupy four of the top five positions; Hussey is the exception at third. England won the series 4-0.

\subsection{Example 3}

Consider the two-match test series between South Africa and India in India, February 2010. The series was tied at 1-1. Tables 7 and 8 summarize the net wicket-taking (bowling and fielding) and batting contributions of the players. To calculate the batting points, we tried both the earlier stated criteria ( $\{$ win $\}$ and $\{$ not lose $\}$ ). However, under the $\{$ win $\}$ criterion, a significant number of batsmen received negative batting points. Therefore, batting contributions are calculated using the $\{$ not lose $\}$ criterion. Wicket-taking contributions are calculated using the $\{$ win $\}$ criterion. Raw unadjusted contributions are shown.

Table 7 summarizes the net batting and net wicket-taking contributions in the first test match. South Africa won this match by an innings and 6 runs. Amla scored 253 runs in the match and received the maximum batting points (0.187). On the other hand, Steyn received the maximum wicket-taking points $(0.878)$ in the match. Note that Sehwag and Tendulkar scored almost the same number of runs but received quite different numbers of points. This is because Tendulkar made 100 runs in the second innings when the defeat of his team was almost certain. Table 8 summarizes the net batting and wicket-taking contributions in the second test. India won the match by an innings and 57 runs. Amla once again scored maximum runs in the match but received fewer batting points than Sehwag and Tendulkar. The Sehwag and Tendulkar partnership was the crucial and decisive one from the point of view of the match result. Laxman and Dhoni also made hundreds in the match but received fewer points than Sehwag and Tendulkar. This is because India were already in a strong position in the match and faced the less pressured situation.

Table 9 shows the total scores over the 2-match series for the top 10 players in rank order, with wicket-taking contributions rescaled so that the total batting and wicket-taking contributions over the series are equal. Harbhajan Singh is placed in top position with maximum total points on the basis of his 7.5 wickets (bowling plus fielding) in the second test; arguably, all these wickets were decisive and played a vital role in the win. Amla scored a massive 490 runs in the two-match series but is some way behind Singh in the adjusted contribution score. Sehwag, who scored 290 runs 
Table 6 Ashes series 2010/2011: Players in rank order of total contribution, batting and wicket taking (bowling and fielding)

\begin{tabular}{|c|c|c|c|c|c|c|c|c|}
\hline Players & Country & $\begin{array}{c}\text { Batting } \\
\text { contribution }\end{array}$ & $\begin{array}{l}\text { Wicket-taking } \\
\text { contribution }\end{array}$ & $\begin{array}{l}\text { Fielding } \\
\text { contribution }\end{array}$ & $\begin{array}{c}\text { Adjusted wicket-taking } \\
\text { contribution }\end{array}$ & $\begin{array}{l}\text { Adjusted fielding } \\
\text { contribution }\end{array}$ & $\begin{array}{l}\text { Total net } \\
\text { contribution }\end{array}$ & Rank \\
\hline Cook & England & 0.885 & 0.000 & 0.106 & 0.000 & 0.059 & 0.944 & 1 \\
\hline Anderson & England & 0.031 & 1.400 & 0.063 & 0.774 & 0.035 & 0.840 & 2 \\
\hline Hussey & Australia & 0.791 & 0.000 & 0.048 & 0.000 & 0.027 & 0.818 & 3 \\
\hline Swann & England & 0.039 & 0.932 & 0.110 & 0.515 & 0.061 & 0.615 & 4 \\
\hline Strauss & England & 0.528 & 0.000 & 0.149 & 0.000 & 0.082 & 0.610 & 5 \\
\hline Tremlett & England & 0.005 & 0.950 & 0.000 & 0.525 & 0.000 & 0.530 & 6 \\
\hline Johnson & Australia & 0.200 & 0.602 & 0.000 & 0.333 & 0.000 & 0.533 & 7 \\
\hline Watson & Australia & 0.434 & 0.107 & 0.077 & 0.059 & 0.043 & 0.536 & 8 \\
\hline Siddle & Australia & 0.089 & 0.491 & 0.002 & 0.271 & 0.001 & 0.361 & 9 \\
\hline Pietersen & England & 0.232 & 0.133 & 0.164 & 0.074 & 0.091 & 0.396 & 10 \\
\hline Finn & England & 0.001 & 0.650 & 0.032 & 0.359 & 0.018 & 0.378 & 11 \\
\hline Prior & England & 0.061 & 0.000 & 0.528 & 0.000 & 0.292 & 0.353 & 12 \\
\hline Haddin & Australia & 0.322 & 0.000 & 0.069 & 0.000 & 0.038 & 0.360 & 13 \\
\hline Trott & England & 0.257 & 0.000 & 0.124 & 0.000 & 0.069 & 0.326 & 14 \\
\hline Bresnan & England & 0.005 & 0.515 & 0.000 & 0.285 & 0.000 & 0.290 & 15 \\
\hline Bell & England & 0.287 & 0.000 & 0.014 & 0.000 & 0.008 & 0.295 & 16 \\
\hline Harris & Australia & 0.010 & 0.426 & 0.000 & 0.235 & 0.000 & 0.245 & 17 \\
\hline Hilfenhaus & Australia & 0.095 & 0.192 & 0.007 & 0.106 & 0.004 & 0.205 & 18 \\
\hline Collingwood & England & 0.059 & 0.075 & 0.156 & 0.041 & 0.086 & 0.187 & 19 \\
\hline Clarke & Australia & 0.163 & 0.000 & 0.010 & 0.000 & 0.006 & 0.169 & 20 \\
\hline
\end{tabular}

Table 7 Raw player contributions for 2010 test series between India and South Africa in India, first test match

\begin{tabular}{|c|c|c|c|c|c|c|c|c|c|}
\hline \multirow[b]{2}{*}{ South Africa } & \multicolumn{2}{|c|}{ Batting contribution } & \multicolumn{2}{|c|}{ Wicket-taking contribution } & \multirow[b]{2}{*}{ India } & \multicolumn{2}{|c|}{ Batting contribution } & \multicolumn{2}{|c|}{ Wicket-taking contribution } \\
\hline & Runs & Points & $\begin{array}{c}\text { Wickets } \\
\text { (bowling }+ \text { fielding) }\end{array}$ & Points & & Runs & Points & $\begin{array}{c}\text { Wickets } \\
\text { (bowling + fielding) }\end{array}$ & Points \\
\hline Smith & 6 & 0.005 & 0.25 & 0.026 & Gambhir & 13 & 0.004 & 0 & 0.000 \\
\hline Boucher (+) & 39 & 0.007 & 0.75 & 0.048 & Vijay & 36 & 0.008 & 0.25 & 0.011 \\
\hline Amla & 253 & 0.187 & 0 & 0.000 & Sehwag & 125 & 0.081 & 0.75 & 0.010 \\
\hline Kallis & 173 & 0.152 & 1 & 0.097 & Tendulkar & 107 & 0.022 & 0 & 0.000 \\
\hline Prince & 0 & 0.000 & 0.25 & 0.026 & Bedrinith & 62 & 0.048 & 0.25 & 0.003 \\
\hline Duminey & 9 & 0.003 & 0.25 & 0.030 & Saha & 36 & -0.023 & 0 & 0.000 \\
\hline de villiers & 53 & 0.030 & 0.25 & 0.023 & Dhoni (+) & 31 & 0.013 & 0.25 & 0.021 \\
\hline Harris & 0 & 0.000 & 3.5 & 0.299 & Mishra & 0 & 0.000 & 0.25 & 0.002 \\
\hline Pernell & 0 & 0.000 & 2.5 & 0.224 & H Singh & 47 & -0.016 & 1.75 & 0.048 \\
\hline Steyn & 0 & 0.000 & 9.25 & 0.878 & Z Khan & 35 & -0.024 & 2.5 & 0.153 \\
\hline Morkel & 0 & 0.000 & 2 & 0.168 & Sharma & 0 & 0.000 & 0 & 0.000 \\
\hline Substitute & 0 & 0.000 & 0 & 0.000 & Substitute & 0 & 0.000 & 0 & 0.000 \\
\hline Extras & 25 & & & & Extras & 60 & & & \\
\hline Match total & 558 & 0.384 & 20 & 1.819 & Match total & 552 & 0.114 & 6 & 0.249 \\
\hline
\end{tabular}

South Africa won by an innings and 6 runs.

in the series, achieved nearly as many adjusted contribution points as Amla. This is because Sehwag's runs came at a more decisive time than Amla's, particularly in the second match of the series. It is quite clear that Amla was the most consistent batsman in the series, but he received fewer points than Harbhajan Singh. This is perhaps because one good bowling spell can change the situation in the match completely and in a very short time; therefore, under the system bowlers can accrue a large number of points. On the other hand, batters take time to make the position strong. This suggests that bowlers are more likely to receive the maximum points, even in the adjusted contribution system.

\section{Rating test match players}

To rate players on the above measures of contribution may not be totally satisfactory. For instance, consider the above stated Example 3, the two-match series between India and South 
Table 8 Raw player contributions for 2010 test series between India and South Africa in India, second test match

\begin{tabular}{|c|c|c|c|c|c|c|c|c|c|}
\hline \multirow[b]{2}{*}{ South Africa } & \multicolumn{2}{|c|}{ Batting contribution } & \multicolumn{2}{|c|}{ Wicket-taking contribution } & \multirow[b]{2}{*}{ India } & \multicolumn{2}{|c|}{ Batting contribution } & \multicolumn{2}{|c|}{ Wicket-taking contribution } \\
\hline & Runs & Points & $\begin{array}{c}\text { Wickets } \\
\text { (bowling + fielding) }\end{array}$ & Points & & Runs & Points & $\begin{array}{c}\text { Wickets } \\
\text { (bowling + fielding) }\end{array}$ & Points \\
\hline Smith & 24 & 0.023 & 0 & 0.000 & Gambhir & 25 & 0.018 & 0 & 0 \\
\hline Petersen & 121 & 0.079 & 0.5 & 0.019 & Vijay & 7 & 0.005 & 0 & 0 \\
\hline Amla & 237 & 0.101 & 0 & 0.000 & Sehwag & 165 & 0.199 & 0 & 0 \\
\hline Kallis & 30 & 0.030 & 0.5 & 0.031 & Tendulkar & 106 & 0.144 & 0 & 0 \\
\hline Prince & 24 & 0.007 & 0.25 & 0.018 & Bedrinith & 1 & 0.001 & 0.25 & 0.023 \\
\hline Duminey & 6 & 0.001 & 0.75 & 0.053 & Laxman & 143 & 0.048 & 0.25 & 0.018 \\
\hline de villiers (+) & 15 & 0.026 & 0.75 & 0.029 & Dhoni (+) & 132 & 0.034 & 1 & 0.073 \\
\hline Harris & 5 & 0.002 & 0.75 & 0.053 & Mishra & 28 & 0.023 & 3.75 & 0.340 \\
\hline Pernell & 34 & 0.032 & 0 & 0.000 & H Singh & 0 & 0.000 & 7.5 & 1.753 \\
\hline Steyn & 6 & 0.011 & 1 & 0.071 & Z Khan & 0 & 0.000 & 4.5 & 0.273 \\
\hline Morkel & 23 & 0.025 & 1.5 & 0.070 & Sharma & 0 & 0.000 & 2.5 & 0.583 \\
\hline substitute & 0 & 0.000 & 0 & 0.000 & Karthik (sub) & 0 & 0.000 & 0.25 & 0.083 \\
\hline Extras & 61 & & & & Extras & 36 & & & \\
\hline Match total & 586 & 0.338 & 6 & 0.344 & Match total & 643 & 0.473 & 20 & 3.146 \\
\hline
\end{tabular}

India won the match by an innings and 57 runs.

Table 9 Total adjusted player contributions for the 2010 test series between India and South Africa in India (top 10 players)

\begin{tabular}{llcrcr}
\hline Country & Player & $\begin{array}{c}\text { Wickets } \\
\text { (bowling }+ \\
\text { fielding) }\end{array}$ & Runs & $\begin{array}{c}\text { Adjusted } \\
\text { contribution } \\
\text { points }\end{array}$ & Rank \\
\hline India & H Singh & 9.25 & 47 & 0.430 & 1 \\
South Africa & Amla & 0 & 490 & 0.288 & 2 \\
India & Sehwag & 0.75 & 290 & 0.282 & 3 \\
South Africa & Steyn & 10.25 & 6 & 0.240 & 4 \\
South Africa & Kallis & 1.5 & 203 & 0.209 & 5 \\
India & Tendulkar & 0 & 213 & 0.167 & 6 \\
India & Sharma & 2.5 & 0 & 0.143 & 7 \\
India & Mishra & 4 & 28 & 0.104 & 8 \\
India & Z Khan & 7 & 35 & 0.101 & 9 \\
South Africa & Pernell & 2.5 & 34 & 0.089 & 10 \\
\hline
\end{tabular}

Africa. First, Amla scored 490 runs in the series at an average of 490 runs per innings and received the maximum batting points (0.288), while Sehwag scored 290 runs in the series at an average of 97 runs per innings and received 0.282 points, nearly as many. There is a large difference between their runs but they received almost the same number of contribution points. This is in part because the contribution points system takes little account of contributions after a point when the win or draw probability of any team is close to unity. Second, in both matches, bowlers stand out in the contribution points table: Steyn in the first test (Table 7) and Harbhajan Singh (Table 8) in the second test. Furthermore, there is the fact that negative points can be achieved, and this appears to be more prevalent for batters. To overcome these problems, it may be sensible to use the contributions as one component of a weighted average rating system. The other component might be the raw runs and wickets in the match.
Such a scheme could be developed in a number of ways. Under one such scheme, the batting, bowling and fielding rating points might be calculated in the following way. Let the batting points of player $i(i=1,2, \ldots, 22)$ under a weighted average rating system in a match be

$$
\left(\frac{\sum_{t=1}^{15} C_{i, t, b a t}}{\sum_{i=1}^{22} \sum_{j=1}^{15} C_{i, t, b a t}}+\frac{r_{i}}{R_{T}}\right) \times \frac{1}{2}
$$

where $r_{i}$ is total runs (raw) scored by player $i$ in the match and $R_{T}$ is the total runs scored by both teams (match total). Note that $t$ is indexing each of the 15 sessions in a match. Let the wickettaking (bowling and fielding) points of player $i$ under this weighted average rating system in the match be

$$
\left(\frac{\sum_{t=1}^{15} C_{i, t, \text { bowl }}}{\sum_{i=1}^{22} \sum_{j=1}^{15} C_{i, t, \text { bowl }}}+\frac{w_{i}}{40}\right) \times \frac{1}{2}
$$

where $w_{i}$ is total wickets share of player $i$ in the match. To standardize the contribution scores under this system, we multiply the net player contribution (sum of the batting, bowling and fielding points) by 1038 - the average score per match, based on all test matches from 1877 to 2007 . In this way, points achieved are put on a 'runs-like' scale. In addition, the maximum batting (runs) and wickets points will be reserved up to 1038 points per match.

To illustrate this rating system, Table 10 summarizes the net contributions of the top 26 players in the 104 test matches in 2010, 2011 and 2012 played by the top eight teams. The top two positions are secured by bowlers. DW Steyn, in the number one spot, scored 381 runs and had a wicket share of 109.25. GP Swann secured second place with 593 runs and a wicket share of 102.75. MJ Clarke in third scored 3120 runs. JH Kallis secured the seventh position with his all-round performance. 
Table 10 Batting, wicket taking and net player contributions (points) for 104 matches in 2010, 2011 and 2012

\begin{tabular}{|c|c|c|c|c|c|c|c|c|c|c|c|}
\hline \multirow[t]{2}{*}{ Player } & \multirow[t]{2}{*}{ Country } & \multicolumn{3}{|c|}{$R a w$} & \multicolumn{3}{|c|}{ Contribution } & \multicolumn{3}{|c|}{ Points } & \multirow[t]{2}{*}{ Rank } \\
\hline & & Runs & Bowling share & Fielding share & Batting & Bowling & Fielding & Batting & Wicket taking & Total & \\
\hline DW Steyn & South Africa & 381 & 109.25 & 3.83 & 0.52 & 6.27 & 0.19 & 396.46 & 3628.10 & 4024.57 & 1 \\
\hline GP Swann & England & 593 & 102.75 & 11.83 & 0.79 & 7.00 & 0.62 & 649.85 & 3247.09 & 3896.94 & 2 \\
\hline MJ Clarke & Australia & 3120 & 10.75 & 12.75 & 3.10 & 0.80 & 0.72 & 3200.41 & 651.23 & 3851.64 & 3 \\
\hline JM Anderson & England & 276 & 100.75 & 7.00 & 0.27 & 5.93 & 0.53 & 270.26 & 3008.72 & 3278.98 & 4 \\
\hline M Morkel & South Africa & 277 & 87.25 & 2.25 & 0.36 & 4.96 & 0.33 & 312.72 & 2894.34 & 3207.06 & 5 \\
\hline HM Amla & South Africa & 2678 & 0.00 & 6.33 & 2.71 & 0.00 & 0.39 & 2859.11 & 217.43 & 3076.54 & 6 \\
\hline JH Kallis & South Africa & 2148 & 20.25 & 11.50 & 1.67 & 1.08 & 0.65 & 2115.72 & 939.87 & 3055.58 & 7 \\
\hline SCJ Broad & England & 794 & 76.75 & 1.50 & 0.97 & 4.77 & 0.04 & 878.90 & 2132.11 & 3011.00 & 8 \\
\hline AN Cook & England & 2664 & 0.00 & 8.08 & 2.83 & 0.00 & 0.51 & 2592.61 & 218.97 & 2811.59 & 9 \\
\hline HMRKB Herath & Sri Lanka & 442 & 88.25 & 3.75 & 0.93 & 3.60 & 0.15 & 555.51 & 2189.52 & 2745.03 & 10 \\
\hline MEK Hussey & Australia & 2317 & 4.00 & 11.00 & 2.58 & 0.32 & 0.57 & 2239.66 & 451.16 & 2690.82 & 11 \\
\hline KC Sangakkara & Sri Lanka & 2336 & 0.00 & 2.50 & 2.42 & 0.00 & 0.45 & 2534.22 & 150.07 & 2684.30 & 12 \\
\hline AB de Villiers & South Africa & 2073 & 0.00 & 14.33 & 2.00 & 0.00 & 0.84 & 2220.27 & 461.68 & 2681.94 & 13 \\
\hline Saeed Ajmal & Pakistan & 191 & 75.00 & 2.25 & 0.23 & 4.75 & 0.11 & 241.17 & 2357.11 & 2598.29 & 14 \\
\hline RT Ponting & Australia & 2234 & 0.00 & 9.00 & 2.80 & 0.00 & 0.47 & 2312.36 & 239.48 & 2551.84 & 15 \\
\hline MJ Prior & England & 1749 & 0.00 & 25.42 & 2.15 & 0.00 & 1.61 & 1799.75 & 729.51 & 2529.26 & 16 \\
\hline GC Smith & South Africa & 1815 & 0.00 & 11.75 & 2.10 & 0.00 & 0.61 & 2079.49 & 443.99 & 2523.48 & 17 \\
\hline KP Pietersen & England & 2383 & 4.75 & 5.50 & 2.48 & 0.14 & 0.34 & 2287.78 & 224.46 & 2512.24 & 18 \\
\hline PM Siddle & Australia & 328 & 65.00 & 3.00 & 0.44 & 4.29 & 0.12 & 412.50 & 2082.17 & 2494.67 & 19 \\
\hline IJL Trott & England & 2177 & 2.00 & 4.75 & 1.90 & 0.52 & 0.30 & 2175.08 & 280.40 & 2455.48 & 20 \\
\hline PP Ojha & India & 80 & 74.25 & 2.25 & 0.06 & 4.70 & 0.12 & 69.32 & 2329.51 & 2398.83 & 21 \\
\hline V Sehwag & India & 2158 & 5.75 & 6.50 & 2.05 & 0.36 & 0.42 & 2010.56 & 373.50 & 2384.06 & 22 \\
\hline VD Philander & South Africa & 267 & 61.75 & 0.75 & 0.46 & 2.68 & 0.05 & 289.09 & 2076.79 & 2365.88 & 23 \\
\hline R Ashwin & India & 567 & 58.50 & 0.75 & 0.54 & 3.63 & 0.03 & 490.39 & 1717.50 & 2207.89 & 24 \\
\hline MS Dhoni & India & 1629 & 0.00 & 24.50 & 1.52 & 0.00 & 1.49 & 1478.99 & 695.85 & 2174.84 & 25 \\
\hline Harbhajan Singh & India & 601 & 48.50 & 2.25 & 0.48 & 4.13 & 0.15 & 508.93 & 1575.95 & 2084.89 & 26 \\
\hline
\end{tabular}

The wicket-keeper batsmen MJ Prior and MS Dhoni were in sixteenth and twenty-fifth position, respectively.

Another potential application of our rating system that may be appealing relates to the selection of man of the match, as the choice is often between a batsman or a bowler and occasionally between a player on the winning team or the losing team. Selecting the highest rated player as man of the match has an objective quality. Counting the instances in which the man of the match is on the winning or losing team as opposed to the highest rated player being on the winning or losing team (Table 11), we can see: (a) in only 2 matches (of the 104 matches in 2010-2012) was the man of the match on the losing team; (b) if the man of the match was awarded to the highest rated player (by our method) then there would have been 17 occasions for which the man of the match was on the losing side. Thus, the table suggests that the subjective choice of man of the match is biased towards winners.

Not only would a man-of-the-match award based on maximum rating points be fairer to losers, but also it brings a better balance between batters, bowlers and all-rounders. Among these 104 matches, the man of the match was a batter, bowler, all-rounder in $51,44,5 \%$ of occasions, respectively. The corresponding figures for the highest rated player are 41, 48, $11 \%$, respectively. Thus, these figures suggest that bowlers play a more decisive role than they are given credit for in man-ofthe-match awards.

A drawback of our rating system is that the total number of points achieved is the same for every match. Thus, for matches
Table $112 \times 2$ cross-classification table for 104 test matches in 2010-2012 played by the top eight teams (30 drawn matches are ignored), classified by actual man of the match (MOM) on winning or losing side and highest rated player on winning or losing side

\begin{tabular}{lccc}
\hline & \multicolumn{3}{c}{ Actual MOM award } \\
\cline { 2 - 4 } & On winning team & On losing team & Total \\
\hline Highest rated player & & & \\
On winning team & 57 & 0 & 57 \\
On losing team & 15 & 2 & 17 \\
Total & 72 & 2 & 74 \\
\hline
\end{tabular}

in which the advantage often changes between the teams, while contribution points will be increased standardization moderates this effect. Thus, the unadjusted contributions method will give greater rewards to players in tight contests - this would seem to be an advantage of this method. To overcome these drawbacks, different components of a weighted average scheme might be combined once total scores on each component are determined for a series of matches. Thus, suppose in a five-match series $r$ runs are scored and $w$ wickets taken, and thus 1 wicket is equivalent to $r / w$ runs. Suppose also that the raw total (over all five matches) contribution points are $n$ for batting and $m$ for wicket taking. Then a possible scheme that equally weights batting and wicket taking, and actual runs scored and wickets 
taken and contributions for each, would be:

$$
T_{i}=\frac{\left(r_{i}+\left(\frac{r}{w}\right) w_{i}+\left(\frac{r}{n}\right) n_{i}+\left(\frac{r}{m}\right) m_{i}\right)}{4}
$$

where $r_{i}, w_{i}, n_{i}$ and $m_{i}$ are the runs scored, wickets taken, raw batting contribution points and raw wicket-taking contribution points of player $i$ in the series, and $T_{i}$ is their total weighted average points scored in the series on a 'run-equivalent' scale. Note again that no adjustment accrues to batsmen for wickets lost or to bowlers for runs conceded.

\section{Discussion}

The purpose of this paper is to evaluate player performances based on batting, bowling and fielding contributions in the match, session by session. We propose a new rating system for bowlers and batters using a common scale. Nominal multinomial logistic regression models are used to model match outcome probabilities for hypothetical and actual positions. Measures of player performance are then calculated using changes in such probabilities session by session. The advantage of looking at the contribution at the end of each session is that we can evaluate the performance of the player taking into account the stage of match in which runs and wicket are earned and conceded. Another advantage of this rating system is that it provides measures (points) for all-rounders, wicket keepers and fielders, again on the same common scale. The ICC rating does not take into account fielding contributions of players. Furthermore, our rating system has the potential to select man of the match, man of the series, best player of the year and new emerging player of the year. These measures could also be helpful for team selectors and management to select a best possible team based on their current performance.

A limitation of this work is that batting contributions and wicket-taking contributions are measured using subtly different criteria: wicket takers are assumed to contribute to winning while run scorers are assumed to contribute to the contrary, that is, to not losing. These different contribution criteria necessitate the rescaling of contributions to a common base. This can be achieved in principle by choosing a relative rescaling factor such that the total run-scoring contributions balance with the total wicket-taking contributions over the 'history' of the game. This would present a challenge for implementation. A more fundamental challenge, however, is that in assigning wicket-taking contributions to fielders (for catches and run-outs) we must subjectively assign a value for the share due to a fielder. If run-scoring and wicket-taking contributions were determined using the same criterion then we might have chosen the fielder's share objectively in the same way. Unfortunately, we cannot resolve two unknowns with a single constraint.

Another limitation of this work is the weakness of the match prediction model. For example, if both the teams lose wickets on the first day of play then selected models are not able to take into account the number of wickets down for the team batting second (Akhtar and Scarf, 2012). Therefore, the bowlers and fielders of the reference team will not be awarded at this stage. The collection of data on more matches would be beneficial to overcome this problem.

In addition, bowlers are not penalized for giving away runs, and fielders are not rewarded for saving runs, or penalized for giving them away through miss-fields. The latter events are not recorded in match scorecards and thus their incorporation requires a different form of data recording. For runs conceded by bowlers, the concept of a hypothetical match position at session-end cannot be extended because hypothetically a bowler could concede an unlimited number of runs during a session. One might instead consider runs conceded relative to certain norms; this is an avenue for further investigation. For example, one might consider the runs conceded by a bowler during a session relative to a figure for runs conceded that is 'loss neutral' over the session.

However, more positively, it is our view that the rating system shows potential for the more reliable assessment of player performances in batting and wicket taking and in comparing performances between these two disciplines. This potential has been shown not just for an individual match but also for a series of matches. The contributions approach might be used in combination with more traditional performance measures, as we illustrate. If the methodology is to be incorporated into or to supplant the existing standard and commercial measures of performance and rankings, further evaluation of the method is necessary, particularly regarding how the measure would perform in the longer term. To achieve this, many more matches need to be analysed.

It is implicit in the system proposed that only those who play can make a contribution, and furthermore that frontline batters and bowlers have a greater opportunity to make a contribution. Thus, the system will not measure who are the best players. Instead, it will measure who played the 'best' cricket. If one were to consider a rating based on contribution rate (per match played) then the best players might emerge, although again, frontline batters and bowlers would tend to be favoured. An average (eg, mean) contribution per match would also tend to favour (and disfavour in equal measure) those who play few matches due to the increasing variability in the mean as the number of observations decreases. However, one could in principle adjust for this by considering the problem of rating as one of estimating player strengths and shrinking the estimated strengths towards an overall mean strength in the manner described, for example, by McHale and Szczepański (2013) in the context of football.

Finally, the contribution measure might be used to determine the top cricketers of all time. It would be fascinating to determine, for example, whether the great all-rounders of the past (eg, Imran Khan, Ian Botham, Garry Sobers) head such a list, and where the best batters (eg, Don Bradman, Brian Lara, Sachin Tendulkar) lie in relation to the best bowlers (eg, Muttiah Muralitharan, Richard Hadlee, Michael Holding). 
Acknowledgements - We are grateful to two referees for their comments; these helped to improve the paper. We would like to thank Mr. Danny Bland (University of Salford), Mr. Abdul Salam and Habibullah Khan (University of Malakand) for helping us in data collection. Finally, thanks to the Higher Education Commission of Pakistan who provided financial support.

\section{References}

Akhtar S (2011). Statistical modelling in test cricket. PhD Thesis, University of Salford, UK.

Akhtar S and Scarf PA (2012). Forecasting test cricket match outcomes in play. International Journal of Forecasting 28(3): 632-643.

ATP (2012). South African Airways ATP Rankings, http://www .atpworldtour.com/Rankings/Singles.aspx, accessed 25 May 2012.

Beaudoin D and Swartz TB (2003). The best batsmen and bowlers in one-day cricket. South African Statistics Journal 37(2): 203-222.

Borooah VK and Mangan JE (2010). The 'Bradman class': An exploration of some issues in the evaluation of batsmen for test matches, 1877-2006. Journal of Quantitative Analysis in Sports 6(3): 1-21.

Brooks RD, Faff RW and Sokulsky D (2002). An ordered response model of test cricket performance. Applied Economics 34: 2353-2365.

Castrol Football (2012). The Castrol Edge Ranking, http://www.youtube .com/castrol, accessed 25 May 2012.

Elo A (1978). The Rating of Chess players, Past and Present. Arco: New York.

EPSN Cricinfo (2010). Test match records, http://www.espncricinfo. com/ci/engine/series/index.html, accessed 25 May 2012.

FAPL (2012). FA Premier League EA Sports Player Performance Index, http://www.premierleague.com/en-gb/players/ea-sports-player-performance-index.html, accessed 25 May 2012.

FIFA (2012). FIFA/Coca-Cola World Ranking, www.fifa.com/world ranking/rankingtable/index.html, accessed 25 May 2012.

Harville D (1980). Predictions for National Football League games via linear model methodology. Journal of the American Statistical Society 75(371): 516-524.

ICC (2011). Reliance Mobile Test Championship, http://www.reliance iccrankings.com/, accessed 28 January 2011.

Johnston MI, Clarke SR and Noble DH (1993). Assessing player performance in one day cricket using dynamic programming. AsiaPacific Journal of Operational Research 10(1): 45-55.

Koning RH (2009). Sport and measurement of competition. De Economist 157(2): 229-249.

Lewis AJ (2005). Towards fairer measure of player performance in oneday cricket. Journal of the Operational Research Society 56(7): 804-815.
Lewis AJ (2008). Extending the range of player-performance measures in one-day cricket. Journal of the Operational Research Society 59(6): 729-742.

McHale I and Szczepański RL (2013). A mixed effects model for identifying goal scoring ability of footballers. Journal of the Royal Statistical Society Series A (Statistics in Society) 177(2): 397-417.

McHale I, Scarf PA and Folker DE (2012). On the development of a soccer player performance rating system for the English Premier League. Interfaces 42(4): 339-351.

Scarf PA and Akhtar S (2011). An analysis of strategy in the first three innings in test cricket: Declaration and the follow-on. Journal of the Operational Research Society 169(11): 1-10.

Scarf PA and McHale I (2005). Ranking football players. Significance 2(2): 54-57.

Scarf PA and Shi X (2005). Modelling match outcomes and decision support for setting a final innings target in test cricket. IMA Journal of Management Mathematics 16(2): 161-178.

Scarf PA, Shi X and Akhtar S (2011a). On the distribution of runs scored and batting strategy in test cricket. Journal of the Royal Statistical Society, Series A 174(2): 471-497.

Scarf PA, Akhtar S and Rasool Z (2011b). Rating players in test match cricket. In: Proceedings of the 3rd International Conference of Mathematics in Sport, (eds. J. Reade, D. Percy and P. Scarf), Salford, UK, pp 208-214.

Stefani R (1977). Football and basketball predictions using least squares. IEEE Transactions on Systems, Man and Cybernetics 7(2): $117-121$.

Stefani R (2011). The methodology of officially recognized international sports rating systems. Journal of Quantitative Analysis in Sports 7(4): 1-22.

Received 7 June 2012; accepted 4 March 2014 after two revisions

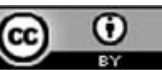

This work is licensed under a Creative Commons Attribution 3.0 Unported License The images or other third party material in this article are included in the article's Creative Commons license, unless indicated otherwise in the credit line; if the material is not included under the Creative Commons license, users will need to obtain permission from the license holder to reproduce the material. To view a copy of this license, visit http://creativecommons .org/licenses/by/3.0/ 